\title{
Subacute thrombosis of left main coronary artery after transcatheter aortic valve replacement
}

\author{
Eiji Ichimoto, Joseph De Gregorio \\ Department of Invasive Cardiology, Englewood Hospital and Medical Center, \\ Englewood, New Jersey, United States
}

An 88-year-old woman was admitted because of presyncope. Echocardiography revealed severe aortic stenosis. Aortic valve area was $0.7 \mathrm{~cm}^{2}$ and mean systolic gradient was $21 \mathrm{~mm} \mathrm{Hg}$. Left ventricular ejection fraction was $50 \%$. Computed tomography (CT) showed calcification in the left main coronary artery (LMCA) (Fig. 1A, B). CT measures were $16 \mathrm{~mm}$ for the distance between the aortic cusp and the left coronary ostium, and $14 \mathrm{~mm}$ for the maximum length of the aortic cusps. Coronary angiography (CAG) described a mild stenosis in the ostial LMCA and a severe stenosis in the mid left anterior descending artery (LAD) (Fig. 1C; Supplementary Video 1 - see journal website).

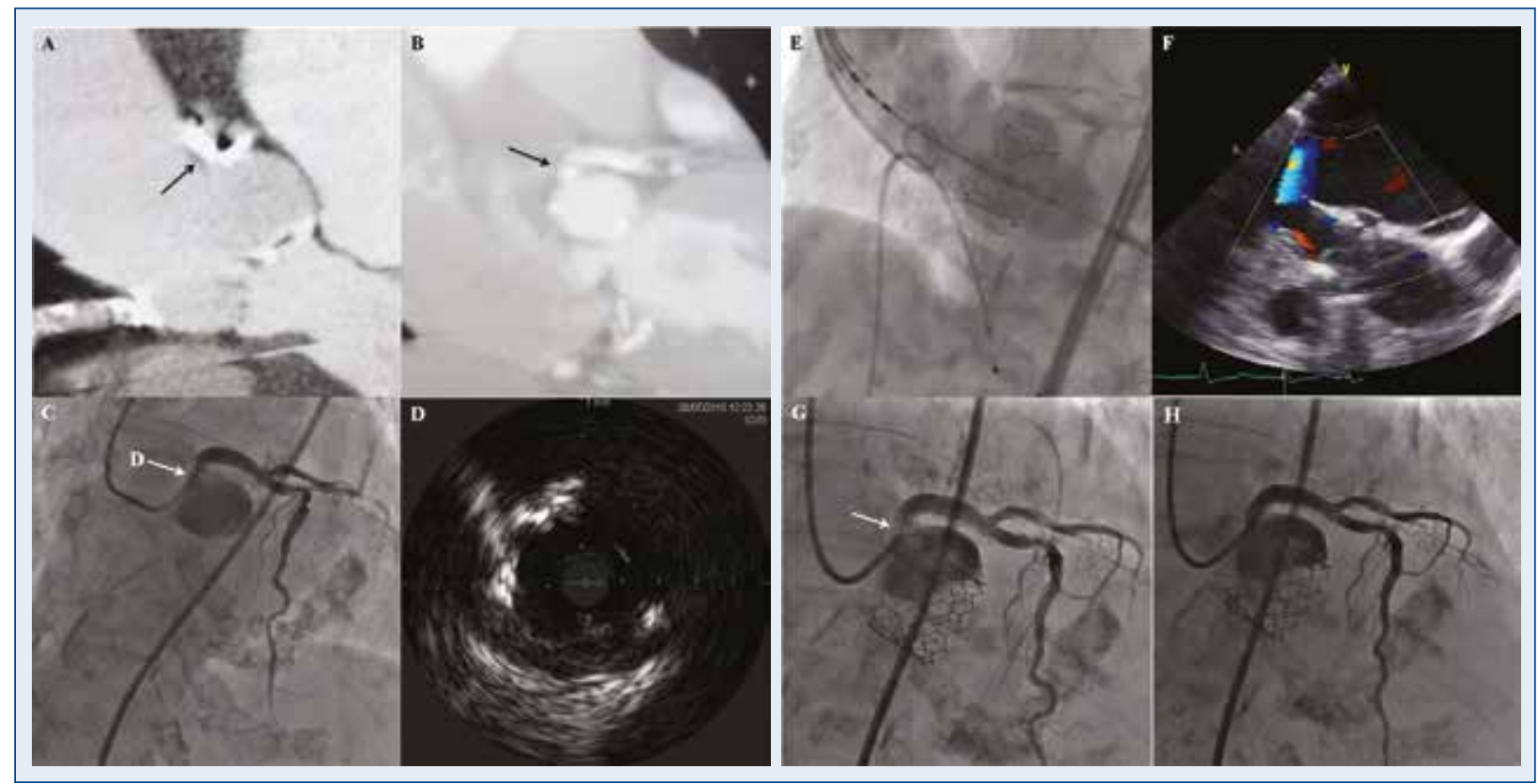

Figure 1. A, B. Computed tomography (CT) before transcatheter aortic valve replacement (TAVR): CT showed calcific atheromatous changes in the aortic valve and calcification in the ostial segment of left main coronary artery (LMCA) indicated by the black arrow; C, D. Coronary angiography and intravascular ultrasound before TAVR: coronary angiography showed a mild stenosis in the ostial segment of LMCA (Supplementary Video 1). Intravascular ultrasound demonstrated calcified and fibrous plaque area; E, F. TAVR procedure and transesophageal echocardiography: supraaortic angiography during TAVR showed a successful deployment of a valve and no occlusion of LMCA. Transesophageal echocardiography showed no aortic dissection or rupture; G, H. Coronary angiography 4 days after TAVR: coronary angiography showed a severe stenosis in the ostial LMCA indicated by the white arrow (Supplementary Video 2). Final coronary angiography after percutaneous coronary intervention demonstrated no significant stenosis of LMCA.

Address for correspondence: Eiji Ichimoto, MD, Department of Invasive Cardiology, Englewood Hospital and Medical Center, 350 Engle Street, Englewood, NJ 07631, USA, tel: +1-201-894-3000, e-mail: e.ichimoto@nifty.com 
Percutaneous coronary intervention (PCI) for the mid LAD was performed. Intravascular ultrasound showed calcified and fibrous plaque area in the ostial LMCA (Fig. 1D). The decision to defer PCI for the ostial LMCA was made. The Society of Thoracic Surgeons score was $6.2 \%$. Subsequently, transcatheter aortic valve replacement (TAVR) was performed and a 23-mm SAPIEN 3 was successfully implanted (Fig. 1E). Transesophageal echocardiography showed no aortic dissection (Fig. 1F). Four days later after TAVR, CAG showed a severe stenosis in the ostial LMCA with chest pain (Fig. 1G; Supplementary Video 2 - see journal website). PCI was performed and an everolimus-eluting stent was successfully implanted (Fig. 1H). Coro- nary obstruction occurs immediately after valve implantation [1]. The present images described a progression of LMCA stenosis 4 days after TAVR. This cause was related to thrombus rather than displacement of calcified native cusp.

Conflict of interest: None declared

\section{Reference}

1. Ribeiro HB, Webb JG, Makkar RR, et al. Predictive factors, management, and clinical outcomes of coronary obstruction following transcatheter aortic valve implantation: insights from a large multicenter registry. J Am Coll Cardiol. 2013; 62(17): 1552-1562, doi: 10.1016/j.jacc.2013.07.040, indexed in Pubmed: 23954337. 